\title{
Erratum: Cosmic ray boosted sub-GeV gravitationally interacting dark matter in direct detection
}

\author{
Wenyu Wang, ${ }^{a}$ Lei Wu, ${ }^{b}$ Jin Min Yang, ${ }^{c, d, e}$ Hang Zhou ${ }^{f}$ and Bin Zhu ${ }^{g, h}$ \\ ${ }^{a}$ Faculty of Science, Beijing University of Technology, \\ Beijing, China \\ ${ }^{b}$ Department of Physics and Institute of Theoretical Physics, Nanjing Normal University, \\ Nanjing 210023, China \\ ${ }^{c}$ CAS Key Laboratory of Theoretical Physics, Institute of Theoretical Physics, \\ Chinese Academy of Sciences, Beijing 100190, China \\ ${ }^{d}$ School of Physical Sciences, University of Chinese Academy of Sciences, \\ Beijing 100049, China \\ ${ }^{e}$ Department of Physics, Tohoku University, \\ Sendai 980-8578, Japan \\ ${ }^{f}$ School of Microelectronics and Control Engineering, Changzhou University, \\ Changzhou, Jiangsu, 213164, China \\ ${ }^{g}$ School of Physics, Yantai University, \\ Yantai 264005, China \\ ${ }^{h}$ Department of Physics, Chung-Ang University, \\ Seoul 06974, Korea \\ E-mail: wywang@bjut.edu.cn, leiwu@njnu.edu.cn, jmyang@itp.ac.cn, \\ zhouhang_hep@126.com, zhubin@mail.nankai.edu.cn
}

ERRATUM TO: JHEP12(2020)072

ArXiv EPrint: 1912.09904

After the submission of the original paper, our co-author Hang Zhou, has changed his affiliation to "School of Microelectronics and Control Engineering, Changzhou University, Changzhou, Jiangsu, 213164, China", and his email address to "zhouhang_hep@126.com". We hereby clarify this situation and make the correction of the author information.

Open Access. This article is distributed under the terms of the Creative Commons Attribution License (CC-BY 4.0), which permits any use, distribution and reproduction in any medium, provided the original author(s) and source are credited. 